\title{
EUROCROP: a concerted action on agricultural research for arable crops competitiveness
}

\author{
Etienne PILORGÉ ${ }^{1}$ \\ Ryszard KOZLOWSKI ${ }^{2}$ \\ Davide VIAGGI ${ }^{3}$ \\ Przemyslaw BARANIECKI ${ }^{2}$ \\ ${ }^{1}$ CETIOM direction scientifique, Grignon, France \\ <pilorge@cetiom.fr> \\ ${ }^{2}$ Institute of Natural Fibres, Poznan, Poland \\ ${ }^{3}$ Department of Agricultural Economics and \\ Engineering, University of Bologna, Bologna, \\ Italy
}

EUROCROP is a coordination action of the $6^{\text {th }}$ PCRD (FP6-2004-SSP-4), involving the stakeholders and actors concerned by arable crops in Europe, which aims to propose research orientations to improve the competitiveness of the sector at the 2015 horizon, i.e. after the next CAP reform. To reach this objective, EUROCROP works at both strategic and scientific levels in order to build common visions of future and research priorities. The EUROCROP project ends in December 2008 and will hold its final conference in Brussels in October 2008 to present its recommendations for arable crops research.

\section{Arable crops competitiveness?}

For EUROCROP, the notion of competitiveness refers to the thinking frame of sustainable agriculture, and includes both economic competitiveness and compliance with environmental and social standards.

\footnotetext{
* The complete results of the project and final recommendations will be presented and discussed in the EUROCROP Conference, next October 2008.

** The EUROCROP conference will be organized in collaboration with the European Economic and Social Committee. It will take place in Brussels, on next $17^{\text {th }}$ October 2008.

www.eurocrop.cetiom.fr.
}

\begin{abstract}
EUROCROP is a coordination action of the $6^{\text {th }}$ Framework Programme (FP6-2004-SSP-4), involving the stakeholders and actors concerned by arable crops in Europe, which aims to propose research orientations to improve the competitiveness of the sector at the 2015 horizon. To reach this objective, EUROCROP developed 4 scenarios for 2015 and examined the main challenges for the arable crops sector. The EUROCROP project ends in December 2008 and will hold its final conference in Brussels in October 2008 to present its recommendations for arable crops research and collect comments.
\end{abstract}

Key words: arable crops, competitiveness, scenarios, research strategy

For its current works, EUROCROP distinguishes two main levels of competitiveness:

1. The economic competitiveness, which refers to a more or less short/medium term approach, with two sub entries:

- economic competitiveness of Arable crops at farm level in EU countries (a crop versus other crops, arable crops versus other land uses) assuming the respect of current regulations when enforced;

- economic competitiveness of EU arable crops on markets: meeting the demand of industries and consumers (as quantities, quality, specifications aspects, regularity of the production, sales prices...).

2. The sustainability and cross compliance which constitute a medium term/ long term approach and rests upon assessment through indicators. This entry focuses on meeting society need, with a higher attention to social and environmental issues.

EUROCROP considers 8 arable crop chains, which received subsidies under the EU Common Agricultural Policies: Cereals (major and secondary), oilseeds crops, sugar-beet, Fibre crops, Potatoes, legumes crops, maize. More widely, it considers arable crops farming systems in their regional and global contexts (figure 1).

The arable crop chains may be considered as value chains whose activity presents positive or negative impacts on horizontal categories which correspond to public goods and involve some major groups of stakeholders (figure 1).

\section{General organisation of the project}

It is the reason why, to meet its goals, EUROCROP uses a cross-cutting approach, first by arable crop (cereals, oilseeds, sugar beets, fibre crops, potatoes, grain legumes and maize), and second by transversal elements of the competitiveness including technical aspects at farming level, farm economics and production costs, outlets and markets, quality of agricultural products, environmental impacts and socio-economic issues (figure 2).

EUROCROP brings together concerned stakeholders and actors, to reach a collective analysis through the elaboration of scenarios and the definition of main stakes and challenges for the arable crops sector. EUROCROP partnership includes organisations using research, including farmers' organisations, and organisations providing research, innovation and extension services. Stakeholders and representative organizations of civil society in the field of environment preservation and consumer advocate are integrated in the partnership of the project, and act especially in the Project Advisory Committee (PADCO).

As shown in the figure 3, EUROCROP works on two interactive levels.

In WP1, a strategic thinking has been developed, using basic foresight approaches at 2015 horizon, in 3 steps:

- Identification of the main challenges to guarantee the AC competitiveness in 2015 future; - Building scenarios for context; 


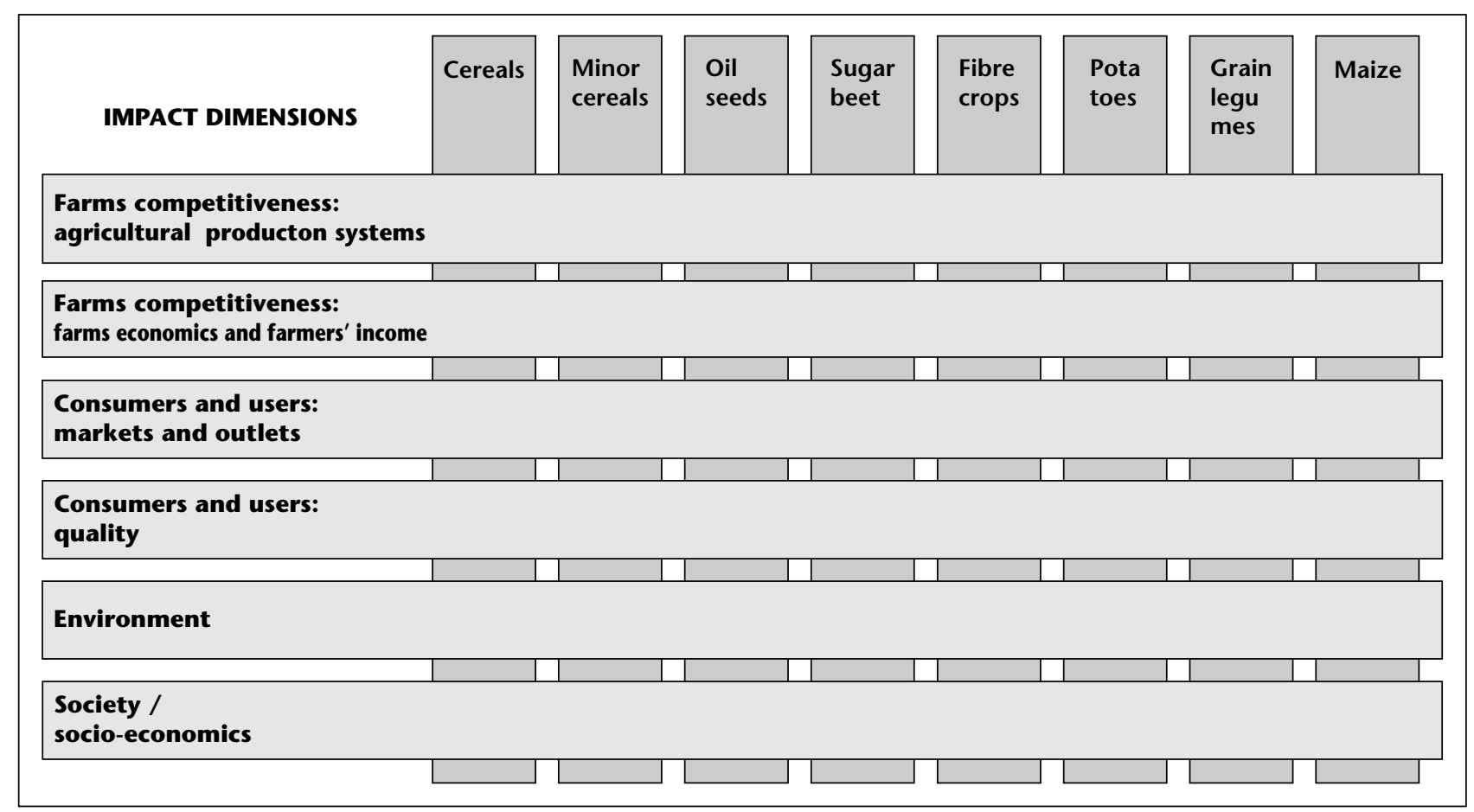

Figure 1. The crop value chains.

- Ranking in priorities the research areas in coherence with the scenarios.

At scientific level (WP2 and WP3) the identification of priority areas have been carried out through:

- a preparatory work on the present research status and knowledge organized to take into consideration; the main challenges and the weaknesses of AC;

- identifying priority research areas and research challenges to fill the gap (experts groups);

- describing briefly the research areas deliverables.

\section{What is the thinking frame for EUROCROP?}

The consensus description of the arable crops context elaborated during the first year of the project could be summarized in the next figure (figure 4), which shows the main dimensions and factors which have to be taken into account to imagine the future challenges to the arable crops sector.

This representation includes 5 external dimensions (Social and economics aspects IN EU, EU outlets and demand/World level markets and trade/Policies and regulations/Environment/ agriculture near context, rural socio economics) and 1 internal dimension. These dimensions are used to identify challenges and elaborate scenarios, constituting "visions of the future", and used to elaborate research strategies.

\section{Preliminary outcome}

The EUROCROP team reached a consensus to consider 4 scenarios, considered as relevant, coherent and plausible. They have no prevision dimension, do not pretend to cover all possibilities, but are only thinking frames used in some kind of "stress studies" allowing to determine what are the challenges which appear to be common to all scenarios, or part of them, and what challenges appear to be specific to a scenario or a situation, thus helping in making priorities. The interest of this approach lays in the fact that it permits to take into account several futures and seems to give chances to avoid critical gaps in making choices and better cover a single but uncertain future. It seems interesting to notice that the scenario which was considered as the most probable at the beginning of the group activity in 2006, is not anymore a tendency in 2008. Making strategies on a unique vision of future may reveal hazardous.

These 4 contrasted scenarios are:

- SC1: "WTO agreement and expensive energy", going on with the liberal world market logics: a WTO agreement is reached, the dominant agricultural model is clearly a productive business agriculture, the CAP is strongly reduced but its orientations are kept, in a context of sustained economic growth.

- SC2: "Europe of regions": no WTO agreement is reached, but the dominant logic remains a liberal one with a double regionalisation: in bilateral agreements and in an increased autonomy of EU regions. The CAP is reduced and decentralized to "regions", on increased Subsidiarity bases. Many agricultural models (led by environment, leisure and tourism, local agro-industries, energy production...), emerge from territorial projects and coexist.

- SC3: "High environmental performance, green Europe": In this scenario, a growing concern about health and environment in opinion becomes a major driver of public policies. The CAP is reoriented to support the "Health \& Environment Performing agriculture" (HEP) model. The context is a low economic growth with no WTO agreement.

- SC4: "challenge of global warming": Global warming becomes sensible and leads the policies. CAP is reoriented to meet the triple necessity to "feed the world", produce energy and manage natural resources. A WTO agreement is reached.

Hypotheses were made on food and biofuels development aspects, and world demand in 


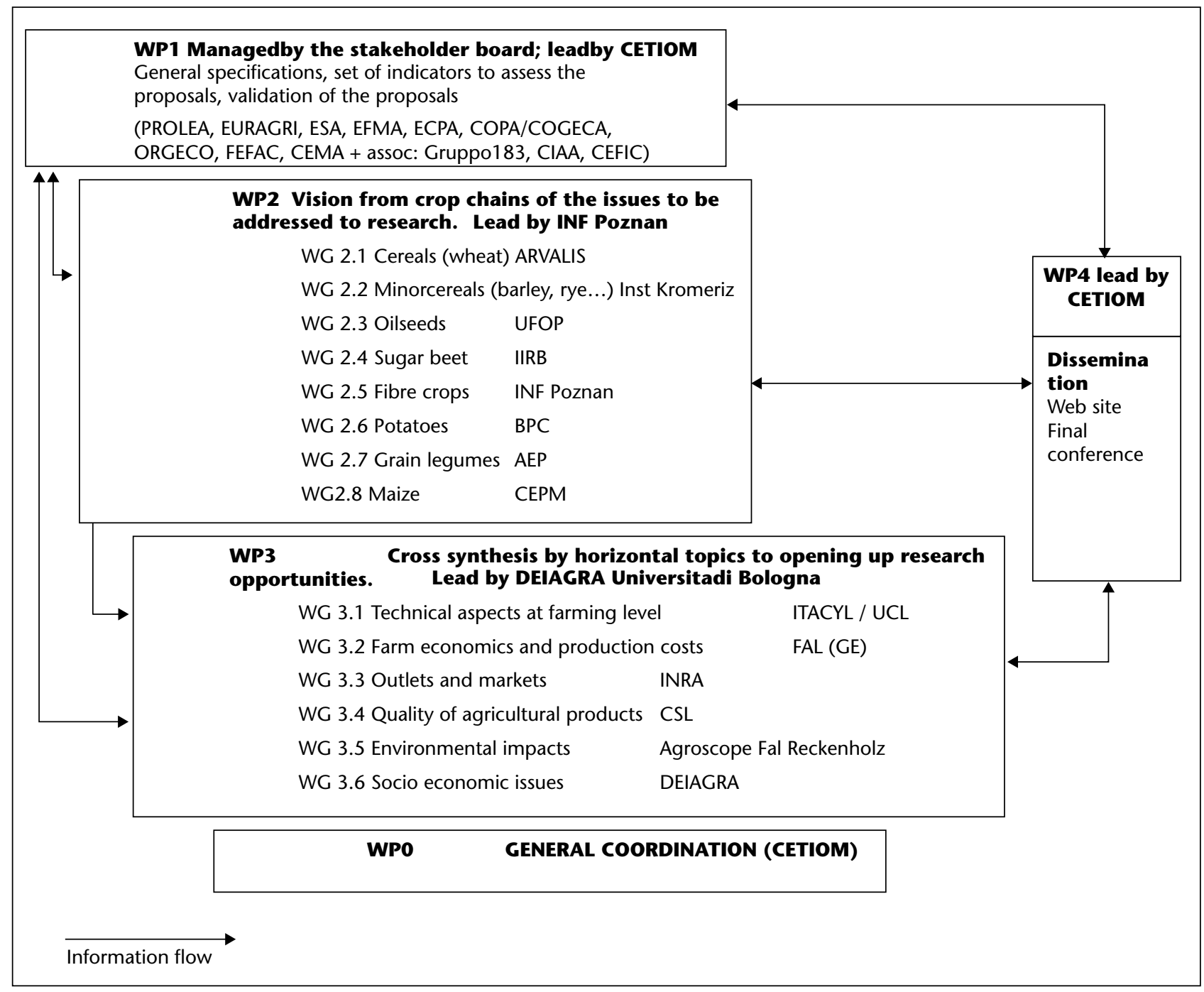

Figure 2. General organisation of the project.

the different scenarios. No scenario considers low costs of energy and/or at the 2015, and consensus has been reached to consider globally high production costs and products prices.

In parallel, the activity of the thematic working groups by crop chains and horizontal issues permitted to identify a number of 36 challenges for arable crops, each of them covering several research goals and topics.

The 36 challenges have been assessed in the frame of each scenario.

At last, 4 of them appear to be a high level priority in the 4 scenarios:

- ensuring food safety;

- improving resource use efficiency: energy;

- improving resource use efficiency: water;

- ensure an effective crop protection in the long term (integrated crop protection).
Four other challenges have a high priority in 3 scenarios at least, or high/medium in the 4 scenarios:

- increase level and stability of yields;

- technical and economic optimisation by innovating sustainable Cropping Systems; - adaptation of production systems and crop rotations according to changes in farming framework conditions;

- improving resource use efficiency: nutrients.

Then other challenges appear common to 2 scenarios with a high level of priority or to 3 with a medium level of priority:

- managing risks for EU farmers;

- developing Non food/ non feed uses;

- maintain and improve soil quality;

- integrating different sustainability concerns

in the design and implementation of innovative cropping systems;
- developing common sustainability assessment methods;

- reinforcing entrepreneurship and innovation capacity of AC systems;

- achieving a positive public perception of arable crops systems.

Three others challenges appear as a priority for one scenario only, but in pole position, and constitute subsidiary challenges, as:

- minimize greenhouse gas emissions per unit of product;

- improving the integration of arable crops into rural territories and economies;

- developing strategies to face climate diversity and climate change.

These results will be refined taking into account the integration level of the different challenges, and comparisons made with current researches or existing recommendations. 


\section{EUROCROP works on two interactive levels.}

\section{C1 POLITICAL / GLOBAL LEVEL} WP1 - PADCO / Core Groupe
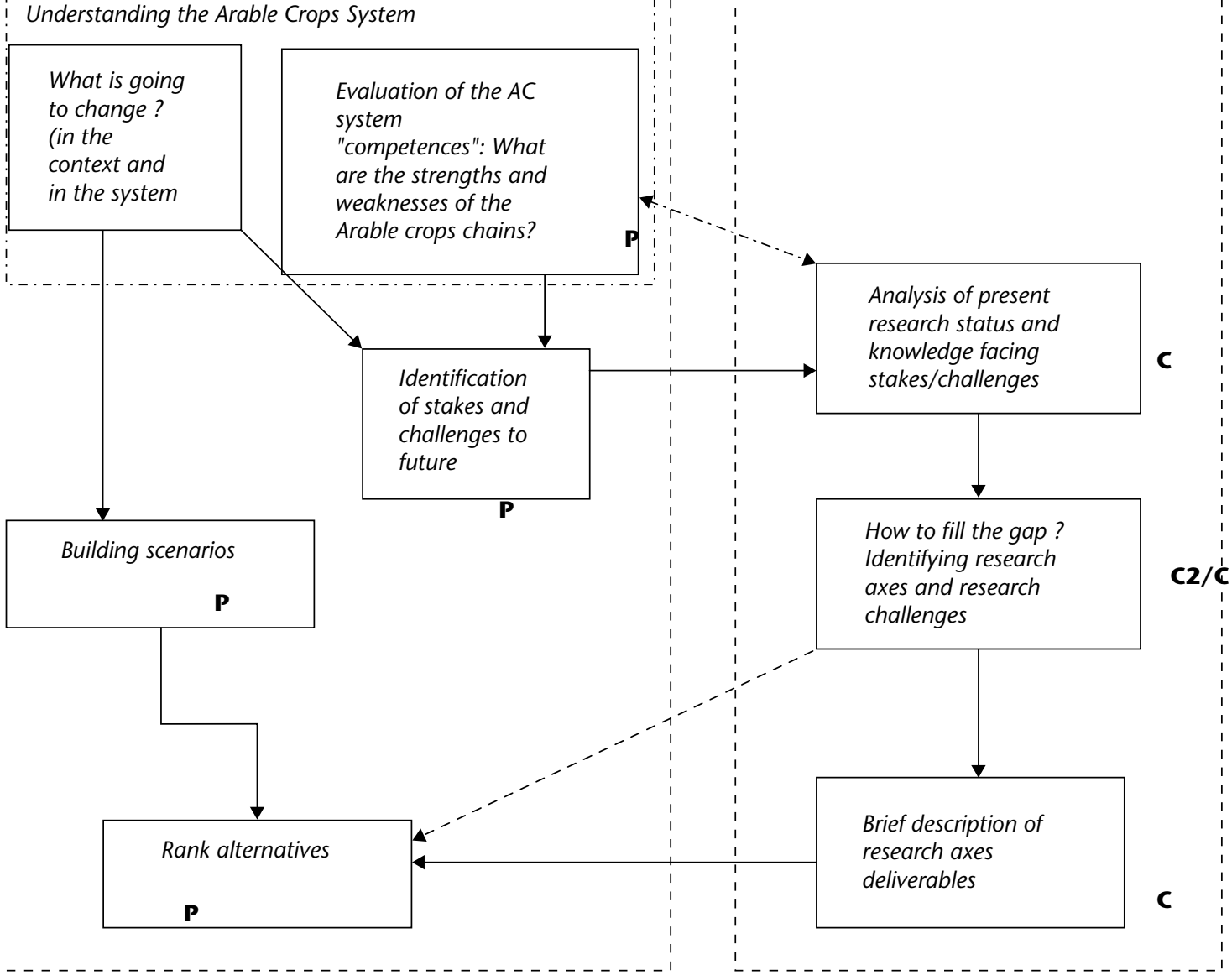

Figure 3. Overview of the methodology tasks organisation. 


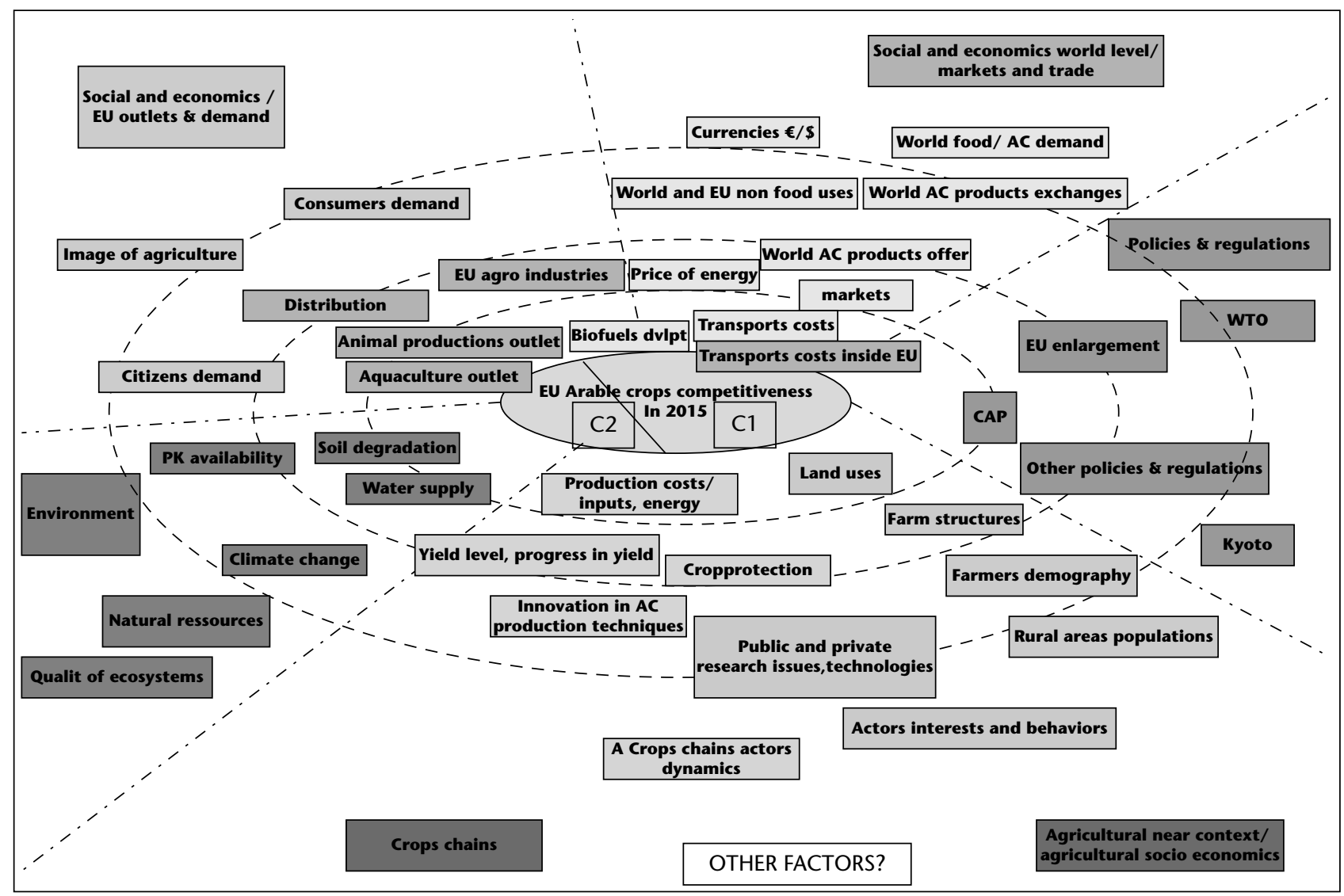

Figure 4. The crops system and its environment.

The working groups also developed detailed descriptions of 66 research topics as answers to the main challenges and goals identified. A dominant feature of the topics identified is the need for flexibility to adapt to changes in the environment (climate), policy and economic conditions. Rather than to specific scenarios, this vision is attached to the idea of a rapidly changing and often unpredictable context.
The developments of commodity markets in recent years, as well as new climate concerns, energy cost and Cap reforms have considerably strengthened such insights.

Difficulties to build a systematic approach to deal with arable crops research derive from the number of interlinkages among issues, from potential different categorisation of the prob- lems and from the large amount of existing research in the field.

Consistent research proposals need to take into account such issues, in particular devising strategies to build new high value information, while at the same time supporting the exploitation of existing research to support the competitiveness of the system. 